\title{
Optimization strategy of mobile data transmission based on optimal crowd feedback
}

Yang Zhang

\begin{abstract}
In order to eliminate the factors that restrict the performance of wireless network data transmission, we proposed the optimal control mechanism of wireless network data transmission. The proposed mechanism solves the intelligence problem of the feedback loop, the mobility of relay nodes, and the feedback of the receiving end. On the one hand, to eliminate the external interference factors, we established an optimal feedback loop control system between the sender and the receiver. On the other hand, in the time linear region, the crowd feedback module is added to the optimal feedback closed-loop control system based on the linear weight. On the basis of the above schemes, we proposed an adaptive optimization model of mobile data transmission. The experiments compared the proposed optimal crowd feedback optimal control scheme with the optimization strategy of the data transmission. From the results of system efficiency and system throughput performance, the proposed optimal crowd feedback optimal control scheme has an obvious advantage.
\end{abstract}

Keywords: Control optimization, Mobility management, Wireless data transmission, Crowd feedback

\section{Introduction}

The diversity of [1] and data services in wireless transmission environment [2] makes the quality of wireless transmission data decrease. Wireless transmission system performance is not stable. These problems seriously affect the quality of the user experience [3]. These issues restrict the way [4] and operating costs of mobile service carriers. In order to solve these problems, the academic and industrial circles have studied the wireless communication protocol [5-8], the wireless data packet $[9,10]$, the transmission performance control optimization [11, 12], and so on.

Jin $M$ proposed a remote wireless transparent transmission protocol for meeting the requirements of the Internet of Things-based intelligent remote monitoring system for construction vehicles on the real time [5]. The distance-sensitive wireless communication protocol was proposed in an article [6] to hearing protectors equipped with in-ear microphones. Chaturvedi A et al. studied the secure wireless communication protocol

Correspondence: yanggzhang@yeah.net

College of International Economics and Trade, Jilin University of Finance and Economics, Jilin, China with the Diffie-Hellman key exchange scheme [7]. The link performance of intra-satellite networking was analyzed and discussed [8] for receiving sensitivity in each model.

The cellular/infostation integrated network was considered by the authors of an article [9] that supports on-demand data service delivery. The novel approaches was proposed in an article [10] that are based on data envelopment analysis (DEA) to further optimize energy consumption in wireless multicast networks.

The random wireless sensor networks were considered, where nodes are distributed randomly and form clusters to transmit the packets to relay clusters using cooperative multi-input-multi-output technique [11]. A simple and effective method is demonstrated to overcome the frequency splitting for an optimal efficiency [12].

The rest of the paper is organized as follows. Section 2 gives the optimal feedback model. Section 3 discussed the optimization strategy of mobile data transmission with crowd feedback. The algorithm analysis results have 


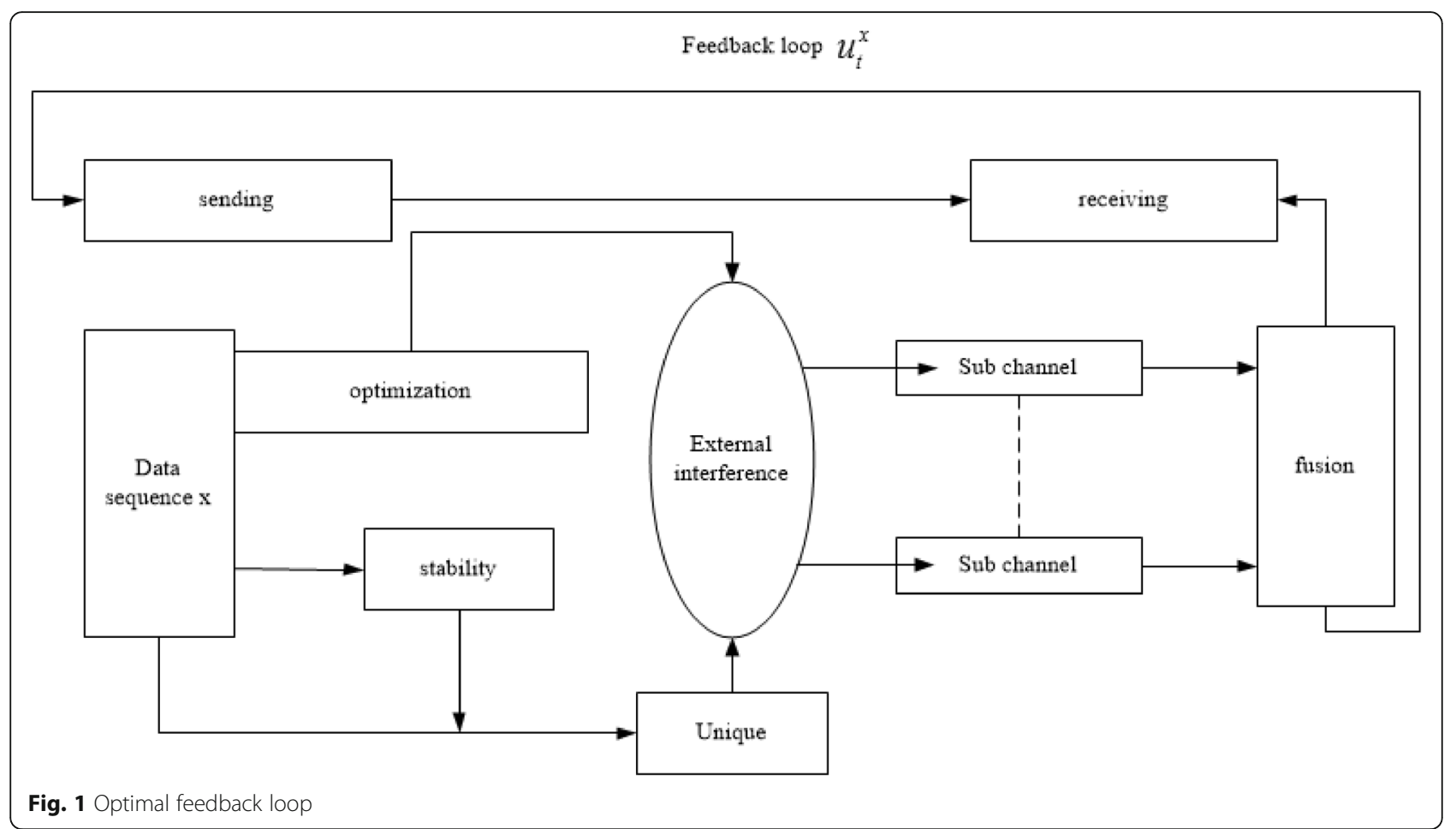

been in Section 4. Finally, the Section 5 concludes this paper.

\section{Optimal feedback model}

The linear data transmission system for wireless communication networks is described as follows:

$$
\left\{\begin{array}{l}
y(x)=H(t) x(t)+F(t) d(s) \\
y(t)=F(x) s
\end{array}\right.
$$

where $x$ said emission signal intensity. $t$ denotes the data transmission time. $H(t)$ said linear vector data transmission. Function $F(t)$ said linear data transmission control vector. $s$ is the linear transmission data sequence. $d(s)$ denotes the size of the data. $y(x)$ indicates the transmitting power signal. $y(t)$ represents the linear time on the total transmit power.

Wireless data transmission is subject to various external disturbances, such as obstacles reflection, building diffraction, ground absorption, and other factors. An optimal feedback loop is formed between the sender and the receiver, which is the key to eliminate the interference factors. The formula (2) describes the optimal feedback closed-loop control systems.

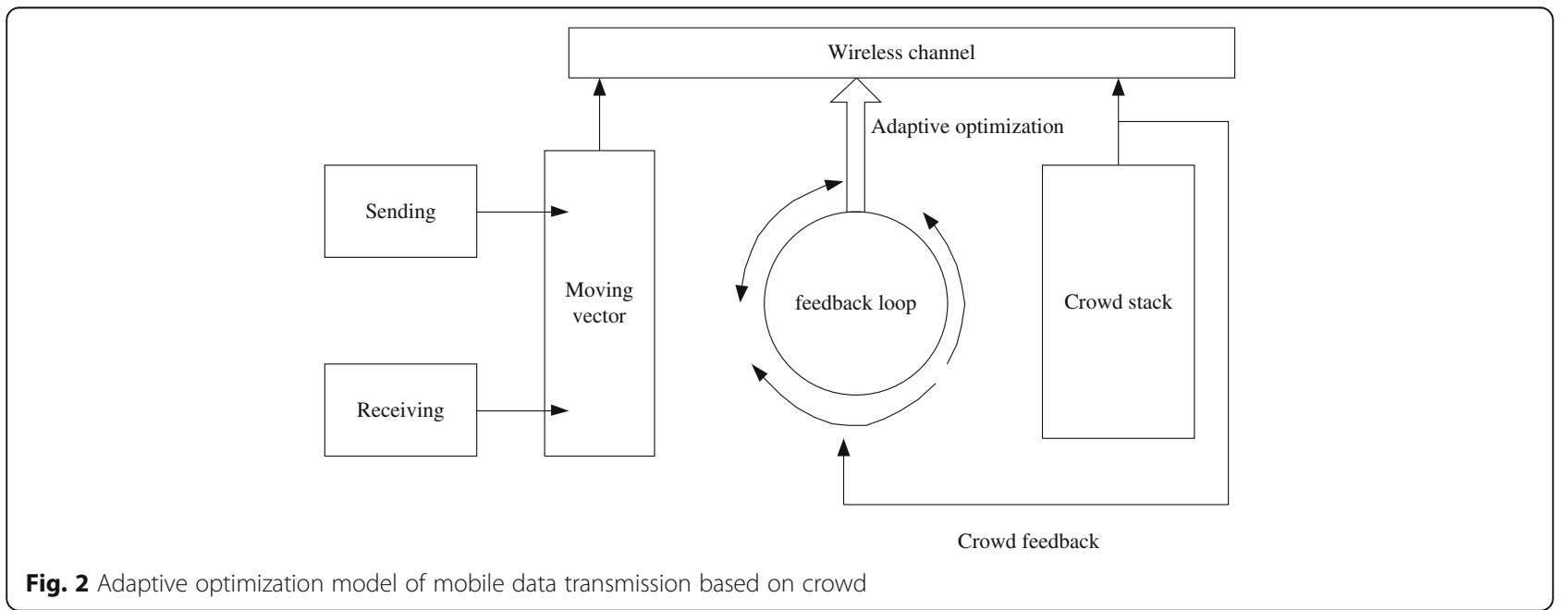




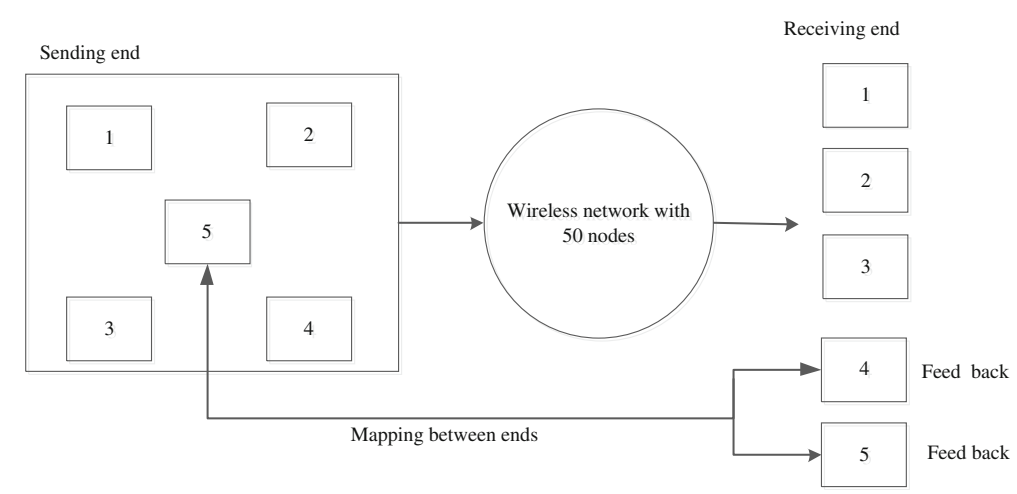

Fig. 3 Experimental architecture

$$
\left\{\begin{array}{l}
P(t)+P(s) H(t)-F(x) y(t) \geq 0 \\
P(x) \leq d(s) \\
\frac{u_{t}^{x}=-H(s)^{-1} F(d)}{\sum_{i=1^{N(x)}} s(i)}
\end{array}\right.
$$

Here, $P(t)$ is the linear data in time domain. $N(x)$ represents the number of symbols in the data sequence $x$. $u_{t}^{x}$ is the feedback loop of the data sequence $x$ in the time domain. The constraint condition $P(t)+P(s) H(t)-$ $F(x) y(t) \geq 0$ can guarantee the stability of wireless data transmission. The constraint condition $P(x) \leq d(s)$ can guarantee the uniqueness and optimality of the closed loop (see Fig. 1).

\section{Optimization strategy of mobile data transmission with crowd feedback}

The optimal feedback closed-loop control system is composed of $m$ data packets. $k$ represents the number of packets. The system transmission efficiency $\eta$ can be obtained from the formula (3).

$$
\left\{\begin{array}{c}
\eta=\frac{\sum_{k=1}^{m} d(k)}{d(s)}, w_{\mathrm{L}}=0 \\
\eta=\frac{\sum_{k=1}^{m} d(k)}{P(x)}, w_{\mathrm{L}}>0
\end{array}\right.
$$

According to the different time domain linear weighting $w_{\mathrm{L}}$, the solution is divided into two kinds. According to the formula (2), it can be known that the optimal system efficiency can be obtained when the $w_{\mathrm{L}}$ is greater than 0 .

In order to keep the probability of $w_{\mathrm{L}}$ be greater than 0 , we design the crowd feedback module in the optimal feedback loop control system. The value feedback vector $x_{\mathrm{CR}}$ can be obtained by the formula (4).

$$
\left\{x_{\mathrm{CR}}=\int_{t \rightarrow T} x(t)+\frac{H(x)-\|F(t)\|}{\sum_{k=1}^{m} d(k)} d(k)=\frac{F d}{P(x)-\frac{1}{2} H(s)^{-1}}\right.
$$

The crowd feedback system efficiency of the transmission after the optimization (see Fig. 2) can be computed with the formula (5).

$$
\eta_{\mathrm{CR}_{\mathrm{OP}}}=\frac{\sum_{k=1}^{m} d(k)}{w_{\mathrm{L}}\|F(x)\|} \cdot \frac{\sqrt{d(x)}}{P(x)}
$$

After optimization, the total length of the data sequence $L_{\text {CR_OP }}$ can be calculated by the formula (6).

$$
L_{\mathrm{CR}}=\left\|\sum_{k=1}^{m} N\left(x_{k}\right)\right\| \frac{w_{\mathrm{L}} \sum_{k=1}^{m} d(k)}{\|F(x)\|^{2}}
$$

\section{Algorithm evaluation results}

The data transmitting end of the experimental platform is a cluster composed of five servers. The receiver runs five Linux virtual systems in a parallel operation. Between the transmitter and the receiver is a wireless communication network composed of 50 mobile nodes. The mapping relationship between the transmitter and two virtual systems of the receiver is an end to end mapping.

Table 1 Experimental settings

\begin{tabular}{llll}
\hline Parameters & Value & Parameters & Value \\
\hline CPU & 8 core & Memory & 32 GB \\
OS & CentOS6.5 & Hard disk space size & 1 TB \\
Driver & Faban-kit & Database & SQL Server \\
Monitor & Vmstat & Number of concurrent session & $1,2,3$ \\
\hline
\end{tabular}




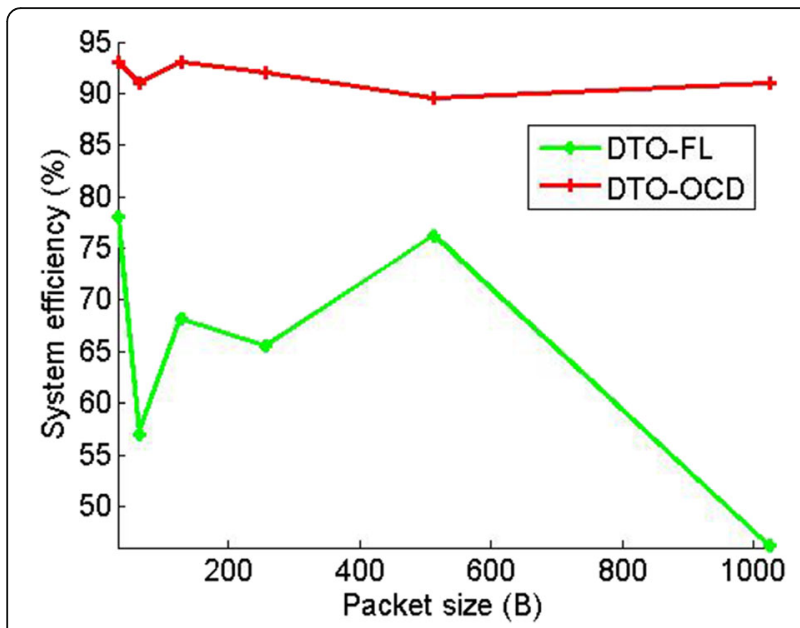

Fig. 4 System efficiency when packet error rate is 0.5\%

The relation is used to establish the optimal crowd feedback closed-loop control system. The experimental structure is shown in Fig. 3. The server hardware and system software configuration is shown in Table 1.

In the experiments, we compared the proposed data transmission optimization-optimal crowd feedback (DTO-OCD) with the data transmission optimization strategy based on the optimized feedback loop data transmission optimization-feedback loop (DTO-FL) with the system efficiency and throughput performance. Figures 4, 5, and 6 give the DTO-OCD and DTO-FL system efficiency with the change of the data packet size when the packet loss rate is $0.5,1$, and $5 \%$, respectively. Found that with the increase of the packet loss rate, the system efficiency of the two mechanisms began to significantly reduce. With the increase of the data packet, the system efficiency of the two mechanisms is

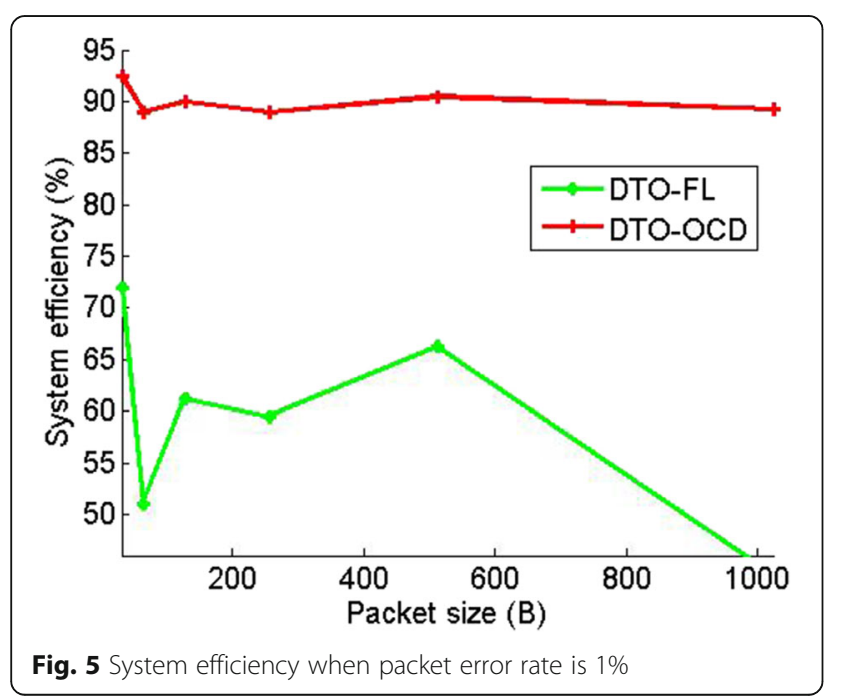

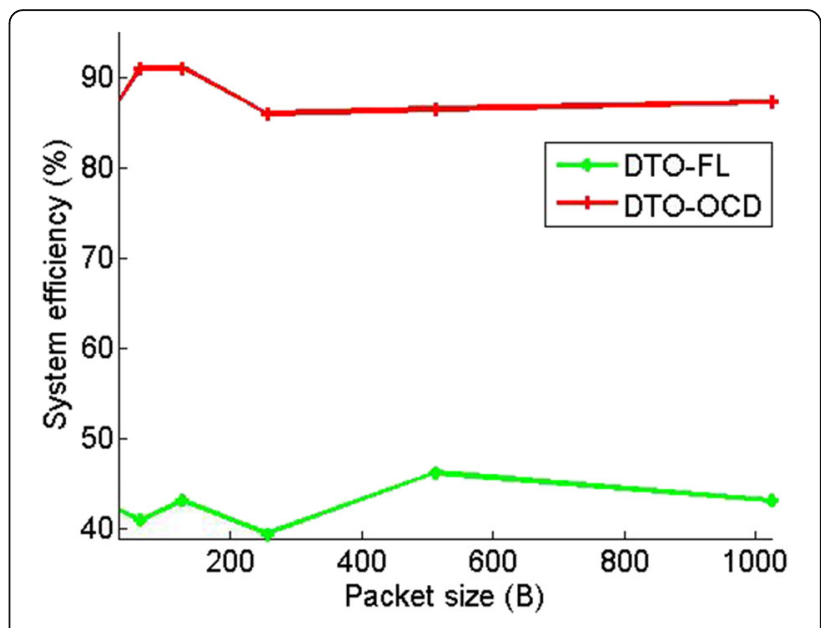

Fig. 6 System efficiency when packet error rate is $5 \%$

chattering. However, the system efficiency of DTO-FL decreased more obviously. Its decline range is the same or two times as much as that of DTO-OCD. DTO-FL jitter is more efficient than that of DTO-OCD.

Figures 7, 8, and 9 give the system throughput rate results with the change of the data packet size when the wireless network mobile speed is $0.5,1$, and $5 \mathrm{~m} / \mathrm{s}$, respectively. With the increase of the speed of node movement, we found that the external disturbance is the key factor to control the quality of data transmission. The system throughput rate of the two mechanisms began to significantly shake. The jitter is especially evident when the moving speed is $5 \mathrm{~m} / \mathrm{s}$. In addition, with the increase of the data package, the system throughput declined. However, the system efficiency of DTO-FL decreased more obviously. The decline range of DTO-FL is 2 times the one of DTO-OCD.

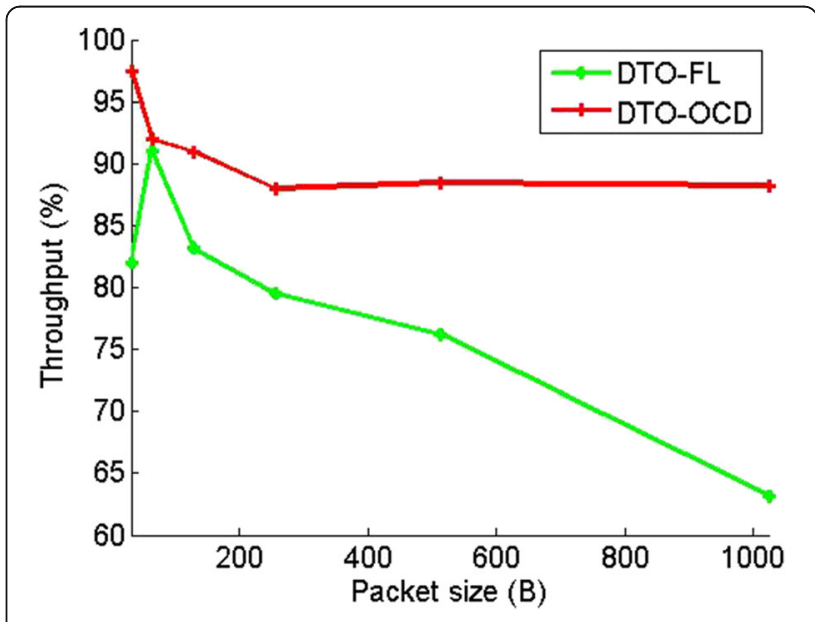

Fig. 7 Throughput when moving speed is $0.5 \mathrm{~m} / \mathrm{s}$ 


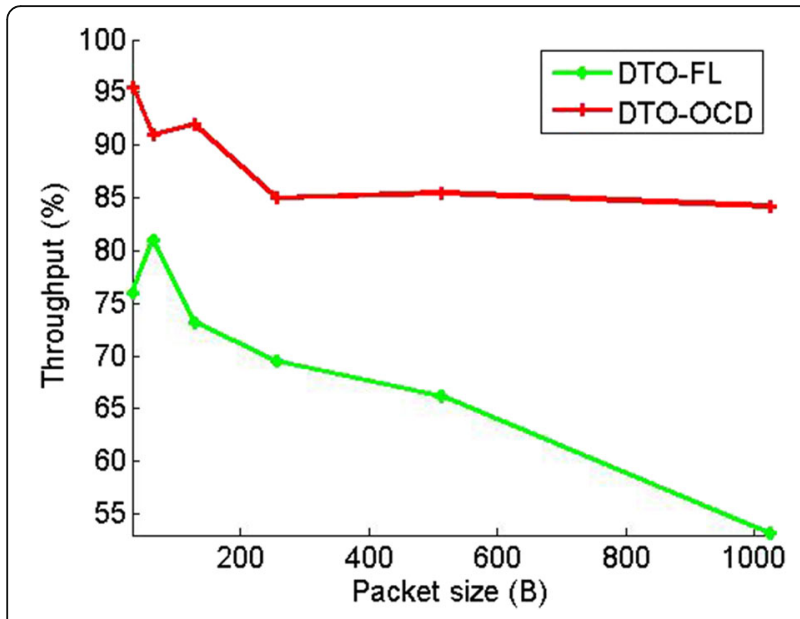

Fig. 8 Throughput when moving speed is $1 \mathrm{~m} / \mathrm{s}$

\section{Conclusions}

Based on the study of the decline reasons of the data transmission performance in the wireless network environment and the influencing factors of the feedback mechanism, we put forward the optimal crowd control of the wireless network data transmission. The innovation of the scheme has also a crowd feedback mechanism, data encapsulation, mobile management, and sensory feedback. Firstly, a closed-loop control system is formed between the transmitter and the receiver, which is affected by the interference of the wireless data transmission. The aim of the system is to eliminate the key factors of these factors. Secondly, according to the time domain linear weight, crowd feedback module is designed to the optimal feedback closed-loop control system. Finally, on the basis of the above researches, we proposed an adaptive optimization model of mobile data

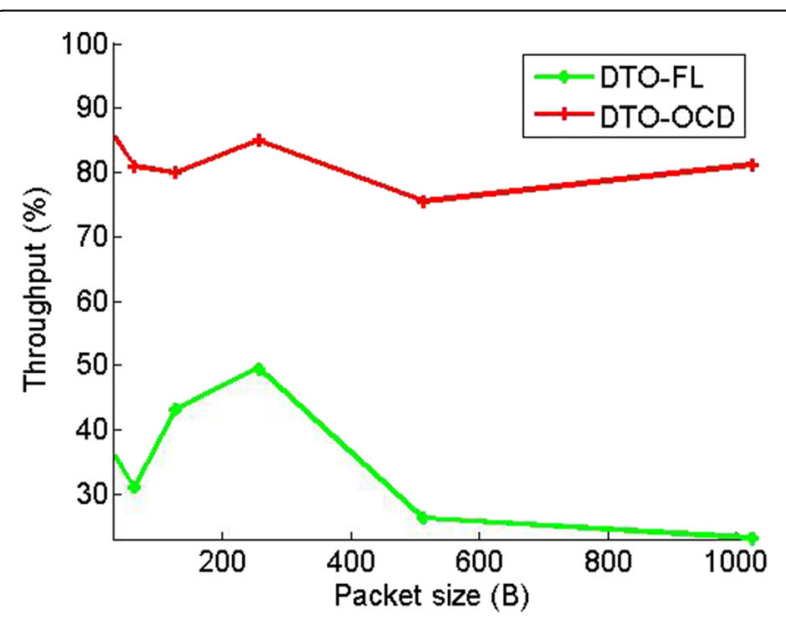

Fig. 9 Throughput when moving speed is $5 \mathrm{~m} / \mathrm{s}$ transmission. Based on the experimental results, we found that the system efficiency and the system throughput rate of the proposed algorithm are higher and the system throughput is higher and smoother.

\section{Competing interests}

The author declares that he/she has no competing interests.

Received: 18 August 2016 Accepted: 11 November 2016

Published online: 09 December 2016

\section{References}

1. Cerpa A, Elson J, Estrin D, et al. Habitat monitoring: application driver for wireless communications technology[J]. Acm Sigcomm Workshop on Data Communications in Latin America \& the Caribbean, 2010;31(2):20-41.

2. Chamotra S, Sehgal R K, Kamal R, et al. Data diversity of a distributed honey net based malware collection system[C]// Emerging Trends in Networks and Computer Communications (ETNCC), 2011 International Conference on. IEEE, 2011:125-129.

3. DM Lestari, D Hardianto, HA Nizar, Analysis of user experience quality on responsive web design from its informative perspective[J]. Int. J. Softw. Eng. Its Appl. 8(5), 53-62 (2014)

4. L Deng, Q Zeng, W Zhou et al., The effect of train formation length and service frequency on the determination of train schedules[J]. Proceedings of the Institution of Mechanical Engineers Part F. J. Rail Rapid Transit. 228(4), 378-388 (2013)

5. M Jin, X Zhou, E Luo et al., Industrial-QoS-oriented remote wireless communication protocol for the internet of construction vehicles[J]. IEEE Trans. Ind. Electron. 62(11), 7103-7113 (2015)

6. REB Serhal, TH Falk, J Voix, Integration of a distance sensitive wireless communication protocol to hearing protectors equipped with in-ear microphones.[J]. J. Acoust. Soc. Am. 133(5), 3273 (2013)

7. A Chaturvedi, N Srivastava, V Shukla, A secure wireless communication protocol using Diffie-Hellman key exchange[J]. Int. J. Comput. Appl. 126, 33-36 (2015)

8. Q Qi, P Li, XU Guo-Dong, Design and simulation of the wireless communication protocol applied to small satellite[J]. LEMCS-14 38(1), 71-76 (2014)

9. T Chen, H Shan, X Wang, Optimal scheduling for wireless on-demand data packet delivery to high-speed trains[J]. IEEE Trans. Veh. Technol. 64(9), 4101$4112(2015)$

10. AA Ajibesin, N Ventura, A Murgu et al., Data envelopment analysis with slacks model for energy efficient multicast over coded packet wireless networks[J]. let Sci. Meas. Technol. 8(6), 408-419 (2014)

11. B Li, H Li, W Wang, Q Yin, H Liu, Performance analysis and optimization for energy-efficient cooperative transmission in random wireless sensor network[J]. IEEE Trans. Wirel. Commun. 12(9), 4647-4657 (2013)

12. J Han, YD Kim, NH Myung, Efficient performance optimisation of wireless power transmission using genetic algorithm[J]. Electron. Lett. 50(6), 462-464 (2014)

\section{Submit your manuscript to a SpringerOpen ${ }^{\circ}$ journal and benefit from:}

- Convenient online submission

- Rigorous peer review

- Immediate publication on acceptance

- Open access: articles freely available online

- High visibility within the field

- Retaining the copyright to your article

Submit your next manuscript at springeropen.com 